\title{
Results from ISTRA+ experiment
}

\author{
Oleg Yushchenko*广 \\ Author affiliation \\ E-mail: Oleg.Yushchenko@cern.ch
}

The form-factor measurements in $K \rightarrow \mu v \gamma$ decay were performed for the first time in the region of INT- term with the total statistics of $22 \mathrm{k}$ decays. The value of $F_{V}-F_{A}=0.16 \pm 0.04$ (stat) \pm 0.05 (syst) was obtained.

The ratio $\operatorname{Br}\left(K_{e 3}\right) / \operatorname{Br}\left(K_{\pi 2}\right)=0.2449 \pm 0.0004 \pm 0.0014$ (syst) was measured with the statistics of $2.2 \mathrm{M}$ events. The value $V_{u s}=0.2275 \pm 0.0009 \pm 0.0022$ (theor) was extracted from these measurements.

35th International Conference of High Energy Physics - ICHEP2010,

July 22-28, 2010

Paris France

\footnotetext{
* Speaker.

${ }^{\dagger}$ On behalf of ISTRA+ Collaboration
} 


\section{1. $K \rightarrow \mu v \gamma$ form-factors}

The photon emission in $K \rightarrow \mu v \gamma$ decay is considered to originate from two processes: bremsstrahlung (IB) and structure-dependent emission ( $\mathrm{SD} \pm$ ) connected with electroweak structure of decay vertex. The last term is very sensitive to EW parameters, provides good test of ChPT and its interference with IB term (INT \pm ) depends on vector and axial form-factors constants (kinematics variables are missed):

$$
\frac{d \Gamma}{d x d y}=A_{I B} f_{I B}+A_{S D}\left[\left(F_{V}+F_{A}\right)^{2} f_{S D+}+\left(F_{V}-F_{A}\right)^{2} f_{S D-}\right]-A_{I N T}\left[\left(F_{V}+F_{A}\right)^{2} f_{I N T+}+\left(F_{V}-F_{A}\right)^{2} f_{I N T-}\right]
$$

The previous measurements by E787 (BNL)[1] and E246 (KEK)[2] were focused on SD \pm region (Fig.1, central plot).
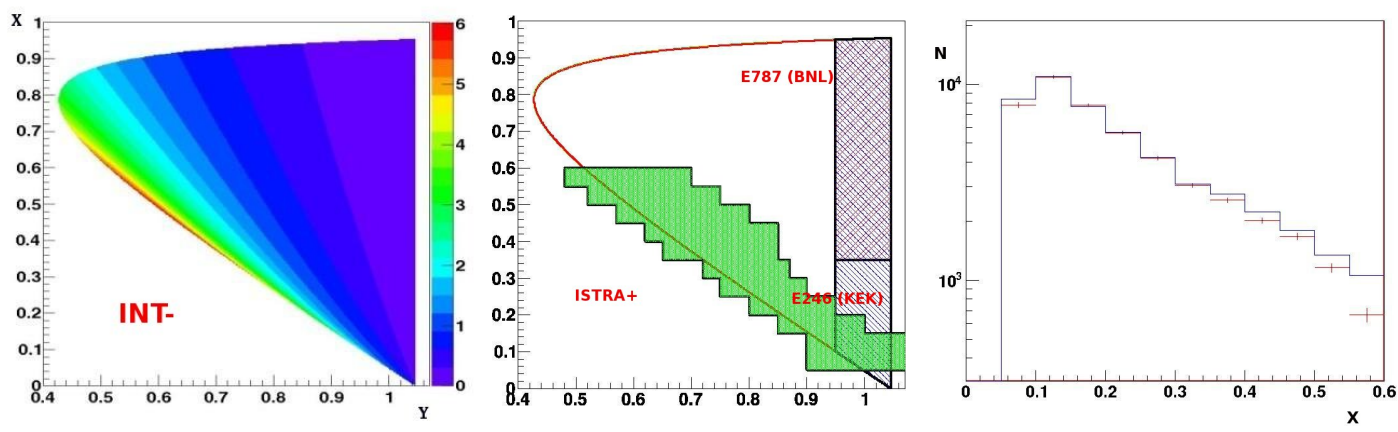

Fig.1 Dalitz plots with INT- term (left) and regions covered by different experiments (center).

Resulting number of signal events extracted in different X-strips (right) with clean IB term (histogram).

The main background sources are $K_{\mu 3}$ and $K_{\pi 2}$ decays which were generated with dedicated $\mathrm{MC}$ with very large statistics in addition to the signal MC.

Three variables $\left(M(\mu v \gamma), Y\right.$ and $\left.\cos \Theta_{\mu \gamma}\right)$ were fitted in separate X-strips to extract number of signal events. In total 22k events were extracted. The results are presented in Fig.1 (right plot). One can clearly observe negative sign of INT- term (lack of events).

Normalizing on IB terms and making use of complete analytical expressions, we got:

$$
F_{V}-F_{A}=0.16 \pm 0.05 \text { (stat) } \pm 0.05 \text { (syst). }
$$

This result can be compared with the prediction of $\mathrm{ChPT} \mathrm{O}\left(p^{4}\right): F_{V}-F_{A}=0.052[3]$.

\section{2. $K \rightarrow e v \pi^{0}$ decay and $V_{u s}$ term of CKM matrix}

This decay provides the best source of information concerning $V_{u s}$ of CKM matrix. Strong interest arose to high statistics/low systematics measurements after E865 (BNL)[4] reported 2.5 $\sigma$ branching increase with respect to PDG value.

The method of measurement is based on the observation that $K \rightarrow e v \pi^{0}$ decay is the dominant source of electrons in kaon decays. All other channels contribute much less than $1 \%$ of electrons. This observation allows to avoid reconstruction of $\pi^{0}$ and use E/P ratio in the calorimeter and $P_{c m}$ for charged track in fit of MC-generated distributions which include the signal as well as main backgrounds. The verification of the method is shown in Fig.2 

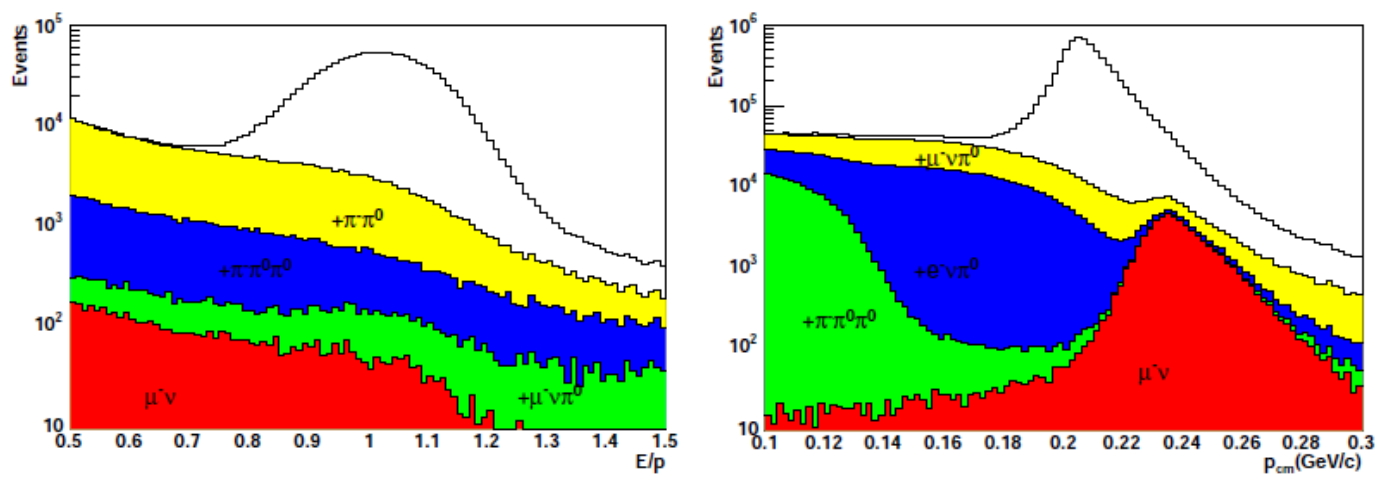

Fig.2 E/P (left) and $P_{c m}$ (right) for different kaon decay channels. The signal is white histogram.

The signal peak was approximated by a sum of two Gaussian and background as simple exponential (for E/P) and as 4-th order polynomial (for $P_{c m}$ ). The total amount of $2.2 \mathrm{M} \mathrm{K} \rightarrow e v \pi^{0}$ events were extracted with

$\operatorname{Br}\left(K_{e 3}\right) / \operatorname{Br}\left(K_{\pi 2}\right)=0.2449 \pm 0.0004$ (stat) \pm 0.0014 (syst), $\quad \operatorname{Br}\left(K_{e 3}\right)=5.124 \pm 0.009$ (stat) \pm 0.030 (syst)\%

$K_{e 3}$ decay rate can be expressed as:

$$
\Gamma\left(K_{e 3}\right)=\frac{\operatorname{Br}\left(K_{e 3}\right)}{\tau(K)}=\frac{G_{\mu}^{2}}{384 \pi^{3}} M_{K}^{5}\left|V_{u s}\right|^{2}\left|f_{+}(0)\right|^{2} I_{K}^{e} S_{E W}\left(1+\delta_{S U_{2}}+\delta_{+}^{e}\right)^{2}
$$

where corrections are absorbed in $S_{E W}\left(1+\delta_{S U_{2}}+\delta_{+}^{e}\right)^{2}$ and decay phase space integral

$$
I_{K}^{e}=\int_{0}^{\left(M_{K}-M_{\pi}\right)^{2}} d t \frac{1}{M_{K}^{8}} \lambda^{3 / 2}\left(f_{+}(t) / f_{+}(0)\right)^{2}, \text { where } \lambda=\left(M_{K}^{2}-t-M_{\pi}^{2}\right)^{2}-4 t M_{\pi}^{2}
$$

contains t-dependent form-factor and is evaluated using out results from [5] where the quadratic non-linearity in the form-factor was measured: $I_{K}^{e}=0.15912 \pm 0.00084 \pm 0.00114$ (syst).

Putting everything together and making use of the theoretical value for $f_{+}(0)=0.961 \pm 0.008$, we get:

$$
\left|V_{u s}\right|\left|f_{+}(0)\right|=0.2186 \pm 0.0009 \pm 0.0012 \text { (theor), } \quad\left|V_{u s}\right|=0.2275 \pm 0.0009 \pm 0.0022 \text { (theor) }
$$

\section{References}

[1] S.C.Adler et al., Phys.Rev.Lett. 85, 2256 (2000).

[2] V.V.Anisimosky et al., Phys.Lett. B562, 166 (2003).

[3] J.Bijnens, G.Ecker and J.Gasser, Nucl.Phys. B396, 81 (1993).

[4] A.Sher et al. (BNL E865) Phys.Rev.Lett. 91, 261802 (2003).

[5] O.P.Yushchenko et al., Phys.Lett. B589 , 111 (2004). 